\title{
Mechanisms of aging-related proteinopathies in Caenorhabditis elegans
}

\author{
Dong-Kyu Kim ${ }^{1,2}$, Tae Ho Kim ${ }^{1,3}$ and Seung-Jae Lee $^{1}$
}

Aging is the most important risk factor for human neurodegenerative diseases such as Alzheimer's and Parkinson's diseases. Pathologically, these diseases are characterized by the deposition of specific protein aggregates in neurons and glia, representing the impairment of neuronal proteostasis. However, the mechanism by which aging affects the proteostasis system and promotes protein aggregation remains largely unknown. The short lifespan and ample genetic resources of Caenorhabditis elegans (C. elegans) have made this species a favorite model organism for aging research, and the development of proteinopathy models in this organism has helped us to understand how aging processes affect protein aggregation and neurodegeneration. Here, we review the recent literature on proteinopathies in $\boldsymbol{C}$. elegans models and discuss the insights we have gained into the mechanisms of how aging processes are integrated into the pathogenesis of various neurodegenerative diseases.

Experimental \& Molecular Medicine (2016) 48, e263; doi:10.1038/emm.2016.109; published online 7 October 2016

\section{INTRODUCTION}

The abnormal deposition of protein aggregates in the form of inclusion bodies is a common pathological feature of most neurodegenerative diseases such as Alzheimer's disease ( $\mathrm{AD})$, Parkinson's disease (PD), Huntington's disease and amyotrophic lateral sclerosis (ALS). AD is typically characterized by the deposition of two types of protein aggregates; one consists of neuritic plaques containing amyloid- $\beta$ (A $\beta$ ) peptides, and the other consists of neurofibrillary tangles containing hyperphosphorylated tau proteins. In addition, AD brains often contain Lewy bodies, intraneuronal inclusion bodies containing $\alpha$-synuclein aggregates. Lewy bodies and the related structures known as Lewy neurites are the pathological hallmarks of PD and dementia with Lewy bodies. Likewise, Huntington's disease is specified by the accumulation of huntingtin aggregates with expanded polyglutamine (polyQ), and ALS is specified by TAR DNA-binding protein 43 (TDP-43) aggregates.

Although polyQ expansion diseases such as Huntington's disease are entirely genetic disorders, most neurodegenerative diseases are sporadic with a few exceptions; $~ 5-10 \%$ of $\mathrm{AD}$ and PD cases show familial inheritance. Mapping of causative gene mutations in these rare cases has been the major driver in the research of neurodegenerative diseases and has provided the rationale for the development of genetic animal models for the diseases. Numerous animal model systems have been established in particular to study the mechanism of protein aggregation and its roles in neurodegeneration. The most widely used in vivo models have been constructed in rodents. Although the rodent models have been very useful in recapitulating some of the major features of neurodegenerative diseases, the results obtained in these models have largely been correlative due to limitations associated with the rodent models, including anatomical complexity and difficulties in genetic modification. In addition, a relatively long incubation period in rodents makes it difficult to assess the role of the aging process in disease pathogenesis.

Aging has long been known as the most important risk factor for neurodegenerative diseases. However, the mechanism as to how aging contributes to the onset of these diseases remains largely speculative. Aging affects many aspects of life sustaining processes, such as energy metabolism, proteostasis and cellular redox control. Elucidating the mechanism underlying the interplay between the aging processes and abnormal protein pathology would be of foremost importance in understanding the pathogenic mechanisms of neurodegenerative diseases.

A nematode species, Caenorhabditis elegans (C. elegans), has been a powerful in vivo model system to study the role of aging processes in the development of neurodegenerative proteinopathies. This model organism has several advantages

\footnotetext{
${ }^{1}$ Department of Biomedical Sciences, Neuroscience Research Institute, Seoul National University College of Medicine, Seoul, Korea; ${ }^{2}$ Department of Biomedical Science and Technology, Konkuk University, Seoul, Korea and ${ }^{3}$ Department of Medicine, Inha University School of Medicine, Incheon, Korea Correspondence: Professor S-J Lee, Department of Biomedical Sciences, Neuroscience Research Institute, Seoul National University College of Medicine, Medical Science Building, Rm B101, 103 Daehak-ro, Jongro-gu, Seoul 03080, Korea.
}

E-mail: sjlee66@snu.ac.kr

Received 16 May 2016; revised 5 July 2016; accepted 12 July 2016 
in studying aging processes and in genetic manipulations. C. elegans has a short lifespan and generation cycle, and its transparent body allows for the visualization of intracellular structures, such as protein aggregates, in real time. In addition, C. elegans has a simple neuronal system of 302 neurons, all of which have been anatomically and developmentally mapped. ${ }^{1}$ Many of genes in C. elegans are homologous to human genes, ${ }^{2}$ including the genes involved in neurodegenerative diseases. ${ }^{3}$ Importantly, several mutant lines with aging phenotypes are available to investigate the role of particular aging processes in proteinopathies. In this study, we review what we have learned from the C. elegans system of the role of aging-related processes in neurodegenerative proteinopathies.

\section{DEGENERATIVE PROTEINOPATHY MODELS IN NEMATODES}

Several transgenic worm models have been developed over the past 20 years. For the modeling of $\mathrm{AD}$, human $\mathrm{A} \beta 42$ was expressed in the body wall muscles by a unc-54 promoter, and these worms exhibited $A \beta$ deposits and progressive motor defects. ${ }^{4}$ Likewise, transgenic worms with pan-neuronal expression of $A \beta$ using the snb-1 promoter showed the accumulation of $A \beta$ aggregates, ${ }^{5,6}$ behavior defects, and shortened lifespan. ${ }^{6}$ These phenotypes were modified with aging. ${ }^{7}$ Transgenic models expressing wild-type or mutant tau (P301L and V337M) under the aex-3 promoter, a pan-neuronal expresser, exhibited neuronal degeneration and presynaptic defects induced by the accumulation of insoluble and phosphorylated tau aggregates. ${ }^{8}$

Transgenic animals expressing human wild-type and mutant forms of $\alpha$-synuclein in neurons exhibited dopaminergic neuronal loss and motor deficits. ${ }^{9-11}$ Recently, a transgenic model for monitoring trans-cellular $\alpha$-synuclein aggregate transmission was generated in C. elegans. These animals showed an age-dependent increase in aggregate transmission, and the rate of aggregate transmission was delayed with anti-aging treatments. ${ }^{12}$

The first C. elegans model for Huntington's disease was generated by expressing a huntingtin fragment containing 150 polyQ repeats in the amphid sensilla of head sensory neurons and resulted in nerve degeneration. ${ }^{13}$ Transgenic expression of expanded polyQ protein appeared to disturb protein-folding homeostasis, ${ }^{14}$ and the worms were protected from the degenerative phenotypes by inducing autophagy. ${ }^{15}$

Familial ALS models have been generated by expressing human superoxide dismutase 1 (SOD1) wild-type or various mutants in the body wall muscles and neurons. These studies consistently showed that the expression of mutant forms of SOD1, but not the wild-type forms, resulted in the accumulation of insoluble SOD1 aggregates, synaptic dysfunction, and motor defects. ${ }^{16-19}$ However, the expression of other ALS-linked genes, wild-type and mutant human TDP-43, and FUS, in neurons using the pan-neuronal snb-1 promoter, also resulted in protein aggregation, neurodegeneration and abnormal motor behavior. ${ }^{20-24}$ Although the expression of the mutants resulted in consistent degenerative phenotypes, effects of wild-type expression varied depending on the study. In addition, loss of function mutants of alfa-1, an orthologue of human C9ORF72, resulted in the degeneration of GABAergic motor neurons and age-dependent motor defects. ${ }^{25}$ Knockdown of $d n c-1$ (the C. elegans homolog of human dynactin 1), another ALS-associated gene, accelerated the development of disease phenotypes including axonal degeneration, abnormal motor symptoms, and reduced lifespan. ${ }^{26}$

\section{HOW AGING PROCESSES AFFECT NEURODEGENERATION}

The availability of various mutant $C$. elegans lines with altered aging processes has allowed the investigation of the mechanism underlying the relationship between aging and neurodegenerative diseases. In this section, we review the aging-related signaling pathways that have been associated with neurodegenerative proteinopathies in C. elegans models.

\section{Insulin/IGF-1-like signaling (IIS)}

The insulin/IGF-1-like signaling (IIS) pathway is a nutrientsensing signaling system and is evolutionarily conserved in higher organisms. ${ }^{27}$ The downregulation of IIS extends lifespan in yeast, Drosophila and mice. ${ }^{28,29}$ Among model organisms, C. elegans has been a powerful model system for the investigation of aging-associated neurodegenerative diseases by regulating the IIS pathway and the related genes. The IIS pathway regulates stress resistance, aging-related genes and protein homeostasis with aging. ${ }^{29}$ The main genetic factors of the C. elegans IIS pathway are the daf- 2 gene, ${ }^{30}$ encoding an insulin and insulin growth factor (IGF-1) receptor, and the daf-16 gene, a forkhead (FOXO) transcription factor. ${ }^{29}$ Under abundant nutrition conditions such as glucose supplementation, the binding of insulin-like peptides to DAF-2 triggers its self-phosphorylation, subsequently resulting in the activation of phosphatidylinositol-3 kinase (AGE-1). ${ }^{31}$ Activated AGE-1 causes the activation of downstream kinases, such as phosphoinositide-dependent kinase 1 (PDK-1) and AKT-1/2 kinase. This signaling cascade promotes the phosphorylation of DAF-16 and inhibits its nuclear localization. The activation of DAF-16 leads to increased longevity, stress resistance and the induction of heat-shock genes such as old-1, sod-3, and small heat-shock protein chaperones. Therefore, the activation of the IIS pathway, which prevents DAF-16 activation, blocks the expression of target genes involved in longevity, metabolism, autophagy and stress resistance. ${ }^{32-34}$ This signaling mechanism explains why mutations in the daf-2 gene extend lifespan, whereas those in daf-16 accelerate aging. ${ }^{35}$

In addition to DAF-16, there are two additional transcription factors, heat-shock factor 1 (HSF-1) and SKN-1/Nrf, acting downstream of the IIS pathway, and these factors are also required for lifespan extension (Figure 1). ${ }^{32,36-39}$ For example, loss of function mutations of $h s f-1$ shortened the lifespan in daf-2 mutant organisms. ${ }^{37,38}$

In addition to the elevated expression of the downstream genes, proteasome activity was also involved in the promotion 


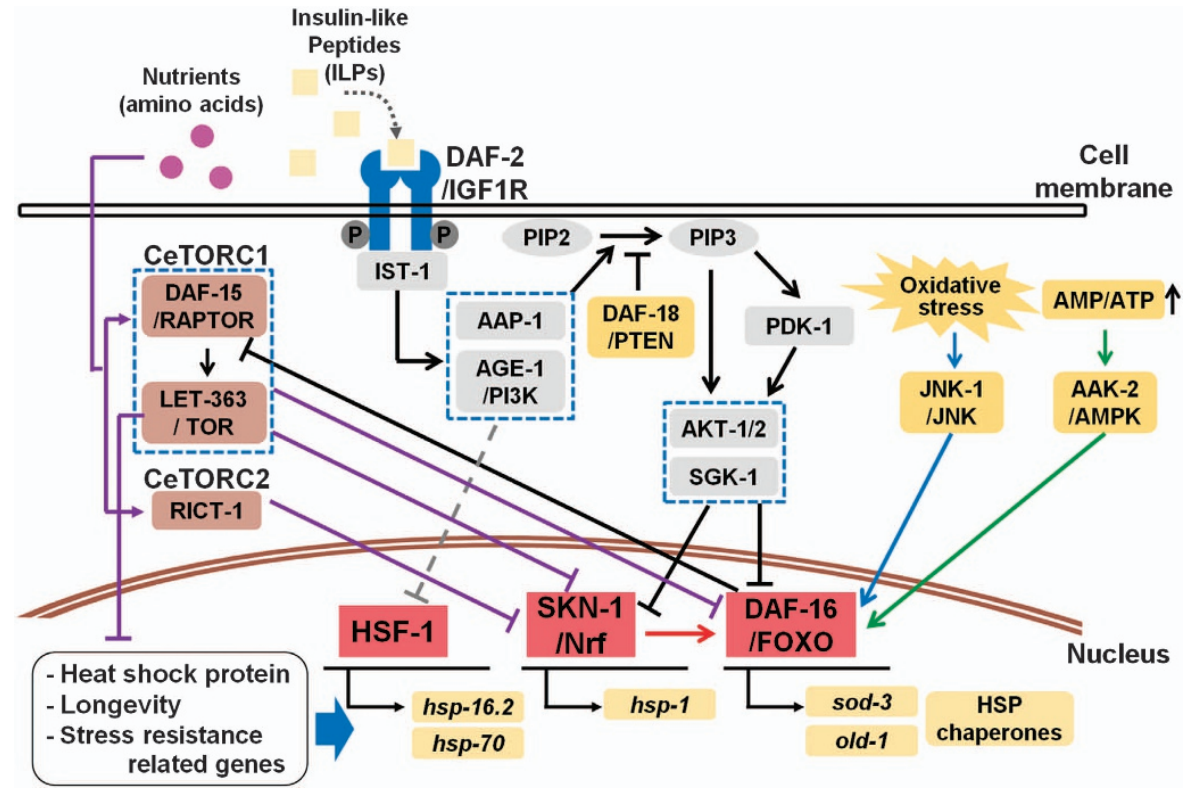

Figure 1 The pathways interacting with insulin/IGF-1 signaling (IIS) in C. elegans. The ILPS, such as INS-1 and INS-7, bind to DAF-2/IGF1R and induce dimerization and phosphorylation, resulting in the recruitment of IST-1, the insulin receptor substrate, to DAF-2, which leads to activation of the phosphoinositide 3-kinases, AGE-1 in neurons and AAP-1 in ubiquitous tissues. Activated AGE-1 leads to the conversion of phosphatidylinositol-4,5-bisphosphate (PIP2) to phosphatidylinositol-3,4,5-trisphosphate (PIP3), subsequently causing a cascade of sequential activation of PDK-1 and AKT-1/AKT-2/SGK-1. These events inhibit the expression of downstream genes that are involved in stress resistance and longevity by inhibiting transcription factors such as DAF-16/FOXO, SKN-1/Nrf and HSF-1. The under abundant nutrients activated CeTORC1 and CeTORC2, which are two different TOR complexes in $C$. elegans, suppress the expression of target genes, whereas the inhibition of daf-15/RAPTOR by the daf-16 gene leads to the induction of the target genes. In particular, CeTORC1 and rict-1/CeTORC2 inhibit the activity of SKN-1, but the expression of the daf-16 gene is regulated by CeTORC1. However, the increased activity of JNK-1 under oxidative stress and the activation of AMPK by high AMP/ATP ratios promote the expression of downstream genes through the induction of the daf-16 gene. The different colors of lines represent the distinct signaling pathways.

of longevity in nematodes. Recent results showed that IIS and proteasome activity were intricately connected. One study showed that in C. elegans, a reduction in the IIS reduced proteasome activity. ${ }^{40}$ However, another study found that IIS reduction increased proteasome activity. ${ }^{41}$ More recently, a study showed that the proteostasis-maintaining mechanism is flexible and capable of responding differentially to distinct challenges. ${ }^{42}$

The role of the IIS pathway in neurodegenerative proteinopathies has been extensively investigated in C. elegans. Transgenic worms expressing human $\mathrm{A} \beta 42$ in the body wall muscles using the unc-54 promoter showed aggregate-mediated toxicity and age-dependent motor defects. ${ }^{43}$ These diseaserelated phenotypes were ameliorated by reducing the activity of the IIS pathway via daf- 2 mutation $^{43}$ or by increasing the expression of heat-shock proteins, ${ }^{44}$ whereas downregulation of the transcription factors DAF-16 or HSF-1 increased aggregation-mediated toxicity. ${ }^{43}$ Activation of $\mathrm{SKN}-1$ also reduced $A \beta$ aggregation and aggregate-induced paralysis in the transgenic worms. ${ }^{45}$ Transgenic worms expressing A $\beta 42$ peptide using the muscle specific myo-3 promoter exhibited an accumulation of autophagosomes, and this phenotype was alleviated by daf- 2 mutations, suggesting the role of autophagy in age-related proteinopathies. ${ }^{46}$
Studies in other proteinopathy models support that the IIS pathway and downstream transcription factors have general roles in the regulation of proteostasis. Transgenic animals generated by crossing long-lived mutant age-1 worms with worms expressing expanded polyQ showed a reduction in age-dependent polyQ aggregation and cellular toxicity. ${ }^{47}$ The induction of DAF-16 and HSF-1 transcription factors and the knockdown of daf-2 also alleviated polyQ-associated proteotoxicity. ${ }^{37,38,47}$ In familial ALS models, mutation of the daf-2 gene mitigated the accumulation of insoluble aggregates and abnormal motor symptoms in transgenic worms expressing $\mathrm{G}^{2} \mathrm{R}^{48}$ or $\mathrm{G} 93 \mathrm{~A}^{19}$ SOD1 mutations. Likewise, mutation of the daf-16 or $h s f-1$ gene in transgenic worms overexpressing the truncated form (TDP-C25) or the full-length human TDP-43 using a pan-neuronal snb-1 promoter resulted in an accumulation of insoluble aggregates and neurotoxicity, whereas daf-2 mutation reduced protein aggregates and alleviated disease phenotypes. ${ }^{22}$

A recent study in worm models of $\alpha$-synuclein transgenic expression showed that IIS regulates the rates of aggregate propagation. In this model, $\alpha$-synuclein aggregate transmission was faster in daf-16 worms than in the wild-type worms, whereas it was slower in daf-2 background worms. $^{12}$ 


\section{Dietary restriction (DR)}

In several model organisms ranging from yeast and flies to rodents and non-human primates, dietary restriction (DR) or caloric restriction resulted in the slowing the aging process. ${ }^{49}$ The effects of DR on the aging process have also been well documented in C. elegans. ${ }^{50}$ Mutations in genes regulating feeding led to defects in pharyngeal muscles in C. elegans, thereby causing DR and a significant extension of lifespan. ${ }^{51}$

The mechanism of how DR regulates longevity remains largely unknown; however, several studies point to the role of reduced oxidative damage and elevated protein turnover. ${ }^{52}$ The overall activity of the protein quality control system declines with age, and this decline is retarded by caloric restriction, resulting in the extension of lifespan. ${ }^{53}$ For example, DR induces autophagy through the inhibition of target of rapamycin (TOR) signaling. ${ }^{54}$ The positive effects of DR on the protein quality control system might explain why DR suppresses protein aggregation and proteotoxicity. ${ }^{55}$

The transcription factors $\mathrm{PHA}-4, \mathrm{SKN}-1$ and $\mathrm{HSF}-1$ regulate glucose metabolism and are required for DR-induced longevity. ${ }^{55-57}$ DR resulted in the activation of these transcription factors and mitigated $\mathrm{A} \beta$ toxicity through this activation. ${ }^{55}$ DR-induced longevity is considered to have complex relationships with multiple distinctive pathways. DR-induced prolonged lifespan required IRE1, one of the endoplasmic reticulum (ER) stress sensors. ${ }^{58}$ In addition, one study showed that loss of pink-1 (an orthologue of human PINK1/PARK6) and $p d r-1$ (an orthologue of human PARKIN/PARK2), PD-linked genes regulating mitophagy, shortened the lifespan of the long-lived mutants, such as daf-2 and eat-2 nematodes, whereas these gene deficiencies had no effect on the wild-type worms. ${ }^{59}$ These results suggest that maintaining ER and mitochondria homeostasis is important for DR-induced longevity and proteostasis.

\section{TOR signaling and autophagy}

The TOR signaling pathway is evolutionarily well-conserved and is a significant player in cellular metabolism, autophagy and aging. ${ }^{60-64}$ Inhibition of the TOR signaling pathway extended the lifespan in C. elegans. ${ }^{65}$ Deletion of let-363/TOR and its binding partner, daf-15/RAPTOR, resulted in an arrest at the L3 larvae stage, and the lifespan was more than double the lifespan of the wild-type organism. ${ }^{66}$

Several downstream events in the TOR signaling pathway might play roles in lifespan extension. One of the mechanisms is through the regulation of mRNA translation. Inhibition of the translation initiation factors ife-2/eIF4E and ifg-1/eIF4G extends lifespan. ${ }^{67-72}$ Autophagy is another downstream event in TOR signaling that regulates the aging process. Inhibition of autophagy by mutations in atg genes, such as atg-7/ATG7, bec-1/ATG6 and atg-18/ATG18, shortened lifespan in C. elegans. ${ }^{73,74}$ Upregulation of the transcription factor HLH-30/TFEB induced autophagic activity and thus increased longevity. ${ }^{75}$ Interestingly, autophagy activation might be the shared mechanism for longevity that is regulated by the IIS, DR and TOR signaling pathways. Autophagy-related genes were upregulated in worms under DR conditions. ${ }^{54}$ Genes that are essential for autophagy, such as bec-1 and vps-34/VPS34, are required for DR-induced longevity. Additionally, IIS inhibition $^{76}$ and TOR inhibition ${ }^{54}$ induced the expression of autophagy-related genes and increased longevity.

Several transcription factors, including $\mathrm{PHA}-4 / \mathrm{FoxA}^{77}$ HSF-1, ${ }^{78}$ SKN-1 ${ }^{65}$ and DAF-16, ${ }^{65,70}$ mediate increased longevity via the inhibition of TOR signaling. These transcription factors are other focal points at which many aging signaling pathways converge. DR caused inhibition of TOR signaling, ${ }^{64}$ which in turn led to activation of autophagy by PHA $-4,{ }^{54}$ an essential transcription factor for DR-induced lifespan extension in worms. ${ }^{56}$ However, not all of these transcription factors have equal roles in the multiple signaling pathways. Unlike the IIS pathway, DAF-16 is not essential for DR-induced longevity, where PHA-4 is the critical factor. ${ }^{56}$

The TOR signaling pathway is a pivotal regulatory mechanism of autophagy, which is the major defense strategy against accumulation of protein aggregates. The role of autophagy in defense against proteinopathies has been extensively studied in transgenic worms expressing expanded polyQ proteins. Knockdown of bec-1, atg-7 and atg-18 caused the accumulation of HTT protein aggregates in transgenic worms expressing HTT-Q150 in muscles and sensory neurons. ${ }^{15}$ In addition, disease phenotypes, such as motor defects and neuronal degeneration, were exacerbated by the inhibition of autophagy. ${ }^{15}$

\section{Mitochondrial respiration}

Mitochondria, the ATP-producing subcellular organelles, have been associated with aging processes. Reduced expression of mitochondrial genes has been linked to lifespan extension in worms. $^{29}$ The mechanisms underlying lifespan extension caused by reduced mitochondrial activity are not fully understood. However, it is likely that the reactive oxygen species produced as byproducts of mitochondrial respiration play important roles. During mitochondrial respiration, the reactive oxygen species are produced by high-energy electrons escaping from the electron transport chain (ETC) and attacking molecular oxygen. Thus, mutations in mitochondrial respiration genes reduced the electron transport in mitochondria and thus dampened reactive oxygen species production. A number of studies have consistently demonstrated that the deficiency of the ETC components increased longevity in C. elegans. ${ }^{79-83}$ Likewise, mutations in $c l k-1$, encoding a mitochondrial protein required for the biosynthesis of ubiquinone, resulted in lifespan extension. ${ }^{84-86}$ In addition, RNAi screening also revealed that the knockdown of genes encoding mitochondrial ETC components slowed the aging rate. ${ }^{87,88}$ Specifically, RNAi knockdown of the complex 1 genes resulted in rescue of metabolite-induced dopamine neurodegeneration. ${ }^{89}$ In contrast, it has also been reported that mutations in gas-1 (general anesthetic sensitive), encoding a subunit of complex I of the ETC, ${ }^{90}$ led to elevated reactive oxygen species production, ${ }^{91}$ and knockdown of the complex II subunit, mev-1, resulted in significant neurodegeneration, ${ }^{89}$ suggesting that the role of 
mitochondrial respiratory function in aging might be contextdependent.

Oxidative stress and mitochondrial dysfunction contribute to the development and progression of many neurodegenerative diseases. ${ }^{92-95}$ Recently, synthetic agents protecting mitochondrial function have been developed and have been shown to be beneficial for neurodegenerative diseases. ${ }^{96,97}$ For example, in the C. elegans AD model, the mitochondrial targeted antioxidant Mito-Q improved the function of the mitochondrial ETC and prolonged lifespan. ${ }^{96}$

Mutant worms expressing truncated PDR-1 protein resulted in an increase in the aggregation of human A53T $\alpha$-synuclein and the degeneration of dopaminergic neurons. ${ }^{98}$ In this study, it was also shown that overexpression of truncated PDR-1 caused changes in mitochondrial membrane potential, suggesting that mitochondrial function might be a target of the pathogenic actions of disease-causing proteins. Similarly, the worms with $p d r-1$ deficiency exhibited increased vulnerability to mitochondrial complex I inhibitors. Mutations in other PD-linked genes, such as $\alpha$-synuclein and DJ-1/PARK7, increased mitochondrial vulnerability to PARKIN. ${ }^{99}$

Similar observations have been made with polyQ proteins. Transgenic worms expressing GFP-tagged polyQ proteins (GFP-HTT28Q or GFP-HTT74Q) in body wall muscle using the unc-45 promoter exhibited increased mitochondrial fragmentation and behavioral defects. ${ }^{100,101}$ Knockdown of $d r p-1$ (dynamin-related protein 1), which regulates mitochondrial fission, ${ }^{102}$ by RNAi alleviated the abnormal motor symptoms in animals expressing expanded polyQ proteins. ${ }^{101}$
Endoplasmic reticulum (ER) protein quality control

Conditions referred to as 'ER stress' often increase the load of misfolded proteins in the ER lumen. ${ }^{103}$ Collective efforts of cells to maintain protein homeostasis in the ER are known as the 'ER protein quality control system.' The expression of various mutant polypeptides or the accumulation of misfolded proteins in the ER triggers an adaptive response, the ER unfolded protein response (UPR), which results in attenuated translation and ER-associated degradation to reduce the unfolded protein load. ${ }^{104}$ However, severe stress conditions lead the UPR signaling to induce cell death by activating pro-apoptotic programs. ${ }^{105,106}$

Although the relationship between the ER stress response and longevity remains poorly understood, several studies have shown that genetic modulation of UPR signaling has modifying effects in animal models of neurodegenerative diseases. Inactivation of inositol-requiring enzyme 1 (IRE-1) and spliced $\mathrm{X}$ box-binding protein 1 (XBP-1), the stress sensors in the UPR signaling pathways, resulted in shortened lifespan in C. elegans. ${ }^{107,108}$ However, in another study, inhibition of the $x b p-1$ pathway in C. elegans also had a protective effect against $\mathrm{A} \beta,{ }^{109}$ suggesting that the role of the ER stress response in neurodegenerative diseases is not simply bimodal.

In an ALS mouse model expressing mutant forms of $\mathrm{Cu} / \mathrm{Zn}$ superoxide dismutase (SOD1), inactivation of BIM, XBP-1, ASK1, PUMA or ATF4 delayed disease onset. ${ }^{110-112}$ In $C$. elegans and zebrafish models, pharmacological induction of elf $2 \alpha$ phosphorylation, representing activation of the protein kinase RNA-like ER kinase (PERK) pathway of the UPR, is protective against TDP-43-induced neurodegeneration. ${ }^{113}$

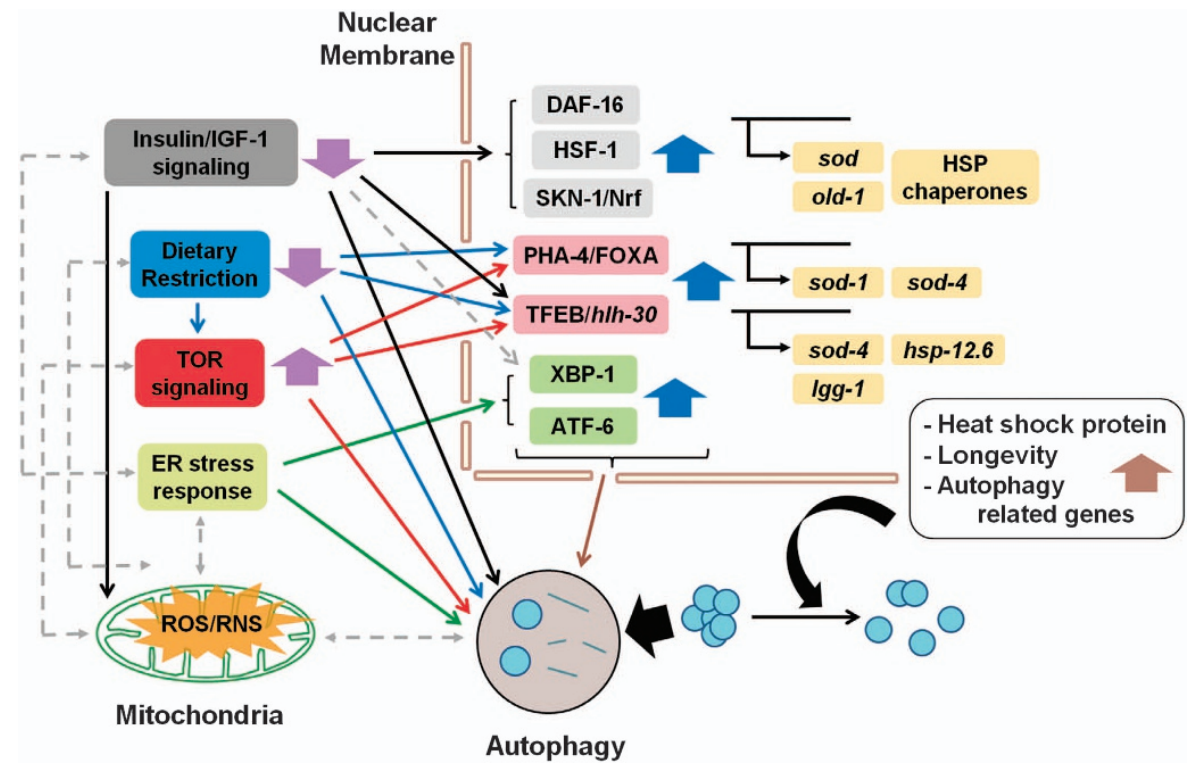

Figure 2 Mechanisms of proteinopathies within the context of the aging processes in C. elegans. The longevity-regulating pathways in worms, including IIS, DR, TOR signaling, autophagy, ER stress response, and mitochondrial respiration pathways, have important roles in protein aggregation by modulating cellular proteostasis through cascades of signaling events that eventually control the transcriptional activities of relevant genes. The colored lines derived from different mechanisms represent the direct or indirect regulation of transcription factors and other signaling pathways by the respective pathways. The dotted gray lines indicate the pathways between which the relationship is suspected but not well established. 
In a PD model, overexpression of XBP-1 had a protective effect against $\alpha$-synuclein-induced dopaminergic neuron degeneration in $C$. elegans, whereas neuron-specific RNAi knockdown of $x b p-1$ accelerated the neurodegeneration process. ${ }^{114}$ Mutations in LRRK2 (PARK8) are responsible for the development of PD. ${ }^{115-117}$ Loss-of-function mutations in C. elegans lrk-1, the orthologue of human LRRK2, caused spontaneous neurodegeneration and hyper-susceptibility to experimental ER stress. ${ }^{118}$ However, LRRK2 protected dopaminergic neurons against neurotoxicity induced by either 6-OHDA or human $\alpha$-synuclein. ${ }^{119}$

A recent study showed that gain-of-function mutations in gfat-1, the key enzyme of the hexosamine pathway, and the metabolites of this pathway, such as $\mathrm{N}$-acetylglucosamine (GlcNAc), induced ER-associated degradation and autophagy, resulting in extended lifespan and protection against a broad spectrum of proteinopathies. ${ }^{120}$ More recently, it has been shown that feeding the worms with GlcNAc delayed the propagation of $\alpha$-synuclein aggregates and thereby slowed down the development of neurodegenerative phenotypes. ${ }^{12}$ Collectively, these genetic studies in worms suggest the close relationships among the ER stress responses, aging and neurodegenerative proteinopathies.

\section{CONCLUDING REMARKS}

Most neurodegenerative diseases are age-related and characterized by proteinopathies. With a short lifespan and wellestablished genetics, C. elegans is an excellent model species to study the relationship between aging and proteinopathies. Figure 2 summarizes the mechanisms of proteinopathymediated neurodegeneration unveiled in C. elegans models. Several signaling pathways, such as the IIS, TOR signaling and ER stress responses, are involved in the regulation of proteinopathies. All of these pathways have roles in the aging processes, and DR controls aging rates through these signaling pathways. To some extent, these aging mechanisms overlap downstream; however, there are also independent components specific to each pathway. How the aging-control signaling network is operated remains the subject of active investigation.

Perhaps the most important lesson we have learned from the studies in C. elegans is that the extent of proteinopathies in disease models is dependent on the organism's aging rate. Genetic modifiers of aging rates change the severity of proteinopathies in such a way that the faster the aging occurs, the more extensive the protein aggregation becomes. Recently, it has been shown that the spread of aggregates was also controlled by aging rates, so that anti-aging treatments can slow the progression of proteinopathies in C. elegans. These findings suggest that the general anti-aging drugs or treatments can be both therapeutic and preventive for neurodegenerative diseases. Future studies of aging signaling and its effects on proteinopathies should uncover drug-sensitive targets for anti-aging and anti-neurodegenerative disease therapeutics.

\section{CONFLICT OF INTEREST}

The authors declare no conflict of interest.

\section{ACKNOWLEDGEMENTS}

This work was supported by a National Research Foundation (NRF) grant funded by the Korean Government (MEST) (NRF-2015R1A2A1A10052540, NRF-2015R1A2A1A15053661), and the Korea Health Technology R\&D Project, Ministry of Health \& Welfare, Republic of Korea (HI14C0093).

1 White JG, Southgate E, Thomson JN, Brenner S. The structure of the nervous system of the nematode Caenorhabditis elegans. Philos Trans $R$ Soc Lond B Biol Sci 1986; 314: 1-340.

2 Lai CH, Chou CY, Ch'ang LY, Liu CS, Lin W. Identification of novel human genes evolutionarily conserved in Caenorhabditis elegans by comparative proteomics. Genome Res 2000; 10: 703-713.

3 Culetto E, Sattelle DB. A role for Caenorhabditis elegans in understanding the function and interactions of human disease genes. Hum Mol Genet 2000; 9: 869-877.

4 Link CD. Expression of human beta-amyloid peptide in transgenic Caenorhabditis elegans. Proc Natl Acad Sci USA 1995; 92: 9368-9372.

5 Link CD. C. elegans models of age-associated neurodegenerative diseases: lessons from transgenic worm models of Alzheimer's disease. Exp Gerontol 2006; 41: 1007-1013.

6 Dosanjh LE, Brown MK, Rao G, Link CD, Luo Y. Behavioral phenotyping of a transgenic Caenorhabditis elegans expressing neuronal amyloid-beta. J Alzheimers Dis 2010; 19: 681-690.

7 McColl G, Roberts BR, Gunn AP, Perez KA, Tew DJ, Masters CL et al. The Caenorhabditis elegans A beta 1-42 model of Alzheimer disease predominantly expresses A beta 3-42. J Biol Chem 2009; 284: 22697-22702.

8 Kraemer BC, Zhang B, Leverenz JB, Thomas JH, Trojanowski JQ, Schellenberg GD. Neurodegeneration and defective neurotransmission in a Caenorhabditis elegans model of tauopathy. Proc Natl Acad Sci USA 2003; 100: 9980-9985.

9 Lakso M, Vartiainen S, Moilanen AM, Sirvio J, Thomas JH, Nass R et al. Dopaminergic neuronal loss and motor deficits in Caenorhabditis elegans overexpressing human alpha-synuclein. J Neurochem 2003; 86: 165-172.

10 Kuwahara T, Koyama A, Gengyo-Ando K, Masuda M, Kowa H, Tsunoda M et al. Familial Parkinson mutant alpha-synuclein causes dopamine neuron dysfunction in transgenic Caenorhabditis elegans. J Biol Chem 2006; 281: 334-340.

11 Hamamichi S, Rivas RN, Knight AL, Cao S, Caldwell KA, Caldwell GA. Hypothesis-based RNAi screening identifies neuroprotective genes in a Parkinson's disease model. Proc Natl Acad Sci USA 2008; 105: 728-733.

12 Kim DK, Lim HS, Kawasaki L, Shim YH, Vaikath N, El-Agnaf OM. et al. Anti-aging treatments slow propagation of synucleinopathy by restoring Iysosomal function. Autophagy (e-pub ahead of print 2 August 2016; doi:10.1080/15548627.2016.1207014).

13 Faber PW, Alter JR, MacDonald ME, Hart AC. Polyglutamine-mediated dysfunction and apoptotic death of a Caenorhabditis elegans sensory neuron. Proc Natl Acad Sci U S A 1999; 96: 179-184.

14 Satyal SH, Schmidt E, Kitagawa K, Sondheimer N, Lindquist S, Kramer JM et al. Polyglutamine aggregates alter protein folding homeostasis in Caenorhabditis elegans. Proc Natl Acad Sci USA 2000; 97: 5750-5755.

15 Jia K, Hart AC, Levine B. Autophagy genes protect against disease caused by polyglutamine expansion proteins in Caenorhabditis elegans. Autophagy 2007; 3: 21-25.

16 Oeda T, Shimohama S, Kitagawa N, Kohno R, Imura T, Shibasaki H et al. Oxidative stress causes abnormal accumulation of familial amyotrophic lateral sclerosis-related mutant SOD1 in transgenic Caenorhabditis elegans. Hum Mol Genet 2001; 10: 2013-2023.

17 Wang J, Farr GW, Hall DH, Li F, Furtak K, Dreier L et al. An ALS-linked mutant SOD1 produces a locomotor defect associated with aggregation and synaptic dysfunction when expressed in neurons of Caenorhabditis elegans. PLoS Genet 2009; 5: e1000350.

18 Gidalevitz T, Krupinski T, Garcia S, Morimoto RI. Destabilizing protein polymorphisms in the genetic background direct phenotypic expression of mutant SOD1 toxicity. PLoS Genet 2009; 5: e1000399. 
19 Li J, Huang KX, Le WD. Establishing a novel C. elegans model to investigate the role of autophagy in amyotrophic lateral sclerosis. Acta Pharmacol Sin 2013; 34: 644-650.

20 Ash PE, Zhang YJ, Roberts CM, Saldi T, Hutter H, Buratti E et al. Neurotoxic effects of TDP-43 overexpression in C. elegans. Hum Mol Genet 2010; 19: 3206-3218.

21 Liachko NF, Guthrie CR, Kraemer BC. Phosphorylation promotes neurotoxicity in a Caenorhabditis elegans model of TDP-43 proteinopathy. J Neurosci 2010; 30: 16208-16219.

22 Zhang T, Mullane PC, Periz G, Wang J. TDP-43 neurotoxicity and protein aggregation modulated by heat shock factor and insulin/IGF-1 signaling. Hum Mol Genet 2011; 20: 1952-1965.

23 Vaccaro A, Tauffenberger A, Aggad D, Rouleau G, Drapeau P, Parker JA. Mutant TDP-43 and FUS cause age-dependent paralysis and neurodegeneration in C. elegans. PLOS ONE 2012; 7: e31321.

24 Vaccaro A, Tauffenberger A, Ash PE, Carlomagno Y, Petrucelli L, Parker JA. TDP-1/TDP-43 regulates stress signaling and age-dependent proteotoxicity in Caenorhabditis elegans. PLoS Genet 2012; 8: e1002806.

25 Therrien M, Rouleau GA, Dion PA, Parker JA. Deletion of C9ORF72 results in motor neuron degeneration and stress sensitivity in $C$. elegans. PLOS ONE 2013; 8: e83450.

26 Ikenaka K, Kawai K, Katsuno M, Huang Z, Jiang YM, Iguchi Y et al. dnc-1/dynactin 1 knockdown disrupts transport of autophagosomes and induces motor neuron degeneration. PLOS ONE 2013; 8: e54511.

27 Barbieri M, Bonafe M, Franceschi C, Paolisso G. Insulin/IGF-I-signaling pathway: an evolutionarily conserved mechanism of longevity from yeast to humans. Am J Physiol Endocrinol Metab 2003; 285: E1064-E1071.

28 Fontana L, Partridge L, Longo VD. Extending healthy life span-from yeast to humans. Science 2010; 328: 321-326.

29 Kenyon CJ. The genetics of ageing. Nature 2010; 464: 504-512.

30 Kimura KD, Tissenbaum HA, Liu Y, Ruvkun G. daf-2, an insulin receptor-like gene that regulates longevity and diapause in Caenorhabditis elegans. Science 1997; 277: 942-946.

31 Morris JZ, Tissenbaum HA, Ruvkun G. A phosphatidylinositol-3-OH kinase family member regulating longevity and diapause in Caenorhabditis elegans. Nature 1996; 382: 536-539.

32 Lin K, Dorman JB, Rodan A, Kenyon C. daf-16: An HNF-3/forkhead family member that can function to double the life-span of Caenorhabditis elegans. Science 1997; 278: 1319-1322.

33 Henderson ST, Johnson TE. daf-16 integrates developmental and environmental inputs to mediate aging in the nematode Caenorhabditis elegans. Curr Biol 2001; 11: 1975-1980.

34 Lee RY, Hench J, Ruvkun G. Regulation of C. elegans DAF-16 and its human ortholog FKHRL1 by the daf-2 insulin-like signaling pathway. Curr Biol 2001; 11: 1950-1957.

35 Kenyon C, Chang J, Gensch E, Rudner A, Tabtiang R. C. elegans mutant that lives twice as long as wild type. Nature 1993; 366: 461-464.

36 Ogg S, Paradis S, Gottlieb S, Patterson GI, Lee L, Tissenbaum HA et al. The Fork head transcription factor DAF-16 transduces insulin-like metabolic and longevity signals in C. elegans. Nature 1997; 389: 994-999.

37 Hsu AL, Murphy CT, Kenyon C. Regulation of aging and age-related disease by DAF-16 and heat-shock factor. Science 2003; 300: $1142-1145$

38 Morley JF, Morimoto RI. Regulation of longevity in Caenorhabditis elegans by heat shock factor and molecular chaperones. Mol Biol Cell 2004; 15: 657-664.

39 Tullet JM, Hertweck M, An JH, Baker J, Hwang JY, Liu S et al. Direct inhibition of the longevity-promoting factor SKN-1 by insulin-like signaling in C. elegans. Cell 2008; 132: 1025-1038.

40 Stout GJ, Stigter EC, Essers PB, Mulder KW, Kolkman A, Snijders DS et al. Insulin/IGF-1-mediated longevity is marked by reduced protein metabolism. Mol Syst Biol 2013; 9: 679.

41 Matilainen O, Arpalahti L, Rantanen V, Hautaniemi S, Holmberg Cl. Insulin/IGF-1 signaling regulates proteasome activity through the deubiquitinating enzyme UBH-4. Cell Rep 2013; 3: 1980-1995.

42 Moll L, Ben-Gedalya T, Reuveni H, Cohen E. The inhibition of IGF-1 signaling promotes proteostasis by enhancing protein aggregation and deposition. FASEB J 2016; 30: 1656-1669.

43 Cohen E, Bieschke J, Perciavalle RM, Kelly JW, Dillin A. Opposing activities protect against age-onset proteotoxicity. Science 2006; 313: $1604-1610$
44 Fonte V, Kipp DR, Yerg J 3rd, Merin D, Forrestal M, Wagner E et al. Suppression of in vivo beta-amyloid peptide toxicity by overexpression of the HSP-16.2 small chaperone protein. J Biol Chem 2008; 283: 784-791.

45 Alavez S, Vantipalli MC, Zucker DJ, Klang IM, Lithgow GJ. Amyloidbinding compounds maintain protein homeostasis during ageing and extend lifespan. Nature 2011; 472: 226-229.

46 Florez-McClure ML, Hohsfield LA, Fonte G, Bealor MT, Link CD. Decreased insulin-receptor signaling promotes the autophagic degradation of beta-amyloid peptide in C. elegans. Autophagy 2007; 3: 569-580.

47 Morley JF, Brignull HR, Weyers JJ, Morimoto RI. The threshold for polyglutamine-expansion protein aggregation and cellular toxicity is dynamic and influenced by aging in Caenorhabditis elegans. Proc Natl Acad Sci USA 2002; 99: 10417-10422.

48 Boccitto M, Lamitina T, Kalb RG. Daf-2 signaling modifies mutant SOD1 toxicity in C. elegans. PLOS ONE 2012; 7: e33494.

49 Sohal RS, Weindruch R. Oxidative stress, caloric restriction, and aging. Science 1996; 273: 59-63.

50 Bishop NA, Guarente L. Genetic links between diet and lifespan shared mechanisms from yeast to humans. Nat Rev Genet 2007; 8: 835-844.

51 Lakowski B, Hekimi S. The genetics of caloric restriction in Caenorhabditis elegans. Proc Natl Acad Sci USA 1998; 95: 13091-13096.

52 Jazwinski SM. Metabolic control and ageing. Trends Genet 2000; 16 : 506-511.

53 Lambert AJ, Merry BJ. Use of primary cultures of rat hepatocytes for the study of ageing and caloric restriction. Exp Gerontol 2000; 35: 583-594.

54 Hansen M, Chandra A, Mitic LL, Onken B, Driscoll M, Kenyon C. A role for autophagy in the extension of lifespan by dietary restriction in $C$. elegans. PLoS Genet 2008; 4: e24.

55 Steinkraus KA, Smith ED, Davis C, Carr D, Pendergrass WR, Sutphin GL et al. Dietary restriction suppresses proteotoxicity and enhances longevity by an hsf-1-dependent mechanism in Caenorhabditis elegans. Aging Cell 2008; 7: 394-404.

56 Panowski SH, Wolff S, Aguilaniu H, Durieux J, Dillin A. PHA-4/Foxa mediates diet-restriction-induced longevity of $C$. elegans. Nature 2007; 447: 550-555.

57 Bishop NA, Guarente L. Two neurons mediate diet-restriction-induced longevity in C. elegans. Nature 2007; 447: 545-549.

58 Taylor RC. Aging and the UPR(ER). Brain Res e-pub ahead of print doi:10.1016/j.brainres.2016.04.017.

59 Sun N, Youle RJ, Finkel T. The mitochondrial basis of aging. Mol Cell 2016; 61: 654-666.

60 Stanfel MN, Shamieh LS, Kaeberlein M, Kennedy BK. The TOR pathway comes of age. Biochim Biophys Acta 2009; 1790: 1067-1074.

61 Kapahi P, Chen D, Rogers AN, Katewa SD, Li PW, Thomas EL et al. With TOR, less is more: a key role for the conserved nutrient-sensing TOR pathway in aging. Cell Metab 2010; 11: 453-465.

62 Evans DS, Kapahi P, Hsueh WC, Kockel L. TOR signaling never gets old: aging, longevity and TORC1 activity. Ageing Res Rev 2011; 10: 225-237.

63 Laplante M, Sabatini DM. mTOR signaling in growth control and disease. Cell 2012; 149: 274-293.

64 Johnson SC, Rabinovitch PS, Kaeberlein M. mTOR is a key modulator of ageing and age-related disease. Nature 2013; 493: 338-345.

65 Robida-Stubbs S, Glover-Cutter K, Lamming DW, Mizunuma M, Narasimhan SD, Neumann-Haefelin E et al. TOR signaling and rapamycin influence longevity by regulating SKN-1/Nrf and DAF-16/FoxO. Cell Metab 2012; 15: 713-724.

66 Vellai T, Takacs-Vellai K, Zhang Y, Kovacs AL, Orosz L, Muller F. Genetics: influence of TOR kinase on lifespan in C. elegans. Nature 2003; 426: 620.

67 Pan KZ, Palter JE, Rogers AN, Olsen A, Chen D, Lithgow GJ et al. Inhibition of mRNA translation extends lifespan in Caenorhabditis elegans. Aging Cell 2007; 6: 111-119.

68 Wang J, Robida-Stubbs S, Tullet JM, Rual JF, Vidal M, Blackwell TK. RNAi screening implicates a SKN-1-dependent transcriptional response in stress resistance and longevity deriving from translation inhibition. PLOS Genet 2010; 6: e1001048.

69 Rogers AN, Chen D, McColl G, Czerwieniec G, Felkey K, Gibson BW et al. Life span extension via elF4G inhibition is mediated by posttranscriptional remodeling of stress response gene expression in C. elegans. Cell Metab 2011; 14: 55-66. 
70 Hansen M, Taubert S, Crawford D, Libina N, Lee SJ, Kenyon C. Lifespan extension by conditions that inhibit translation in Caenorhabditis elegans. Aging Cell 2007; 6: 95-110.

71 Syntichaki P, Troulinaki K, Tavernarakis N. elF4E function in somatic cells modulates ageing in Caenorhabditis elegans. Nature 2007; 445: 922-926.

72 Curran SP, Ruvkun G. Lifespan regulation by evolutionarily conserved genes essential for viability. PLoS Genet 2007; 3: e56.

73 Hars ES, Qi H, Ryazanov AG, Jin S, Cai L, Hu C et al. Autophagy regulates ageing in C. elegans. Autophagy 2007; 3: 93-95.

74 Toth ML, Sigmond T, Borsos E, Barna J, Erdelyi P, Takacs-Vellai K et al. Longevity pathways converge on autophagy genes to regulate life span in Caenorhabditis elegans. Autophagy 2008; 4: 330-338.

75 Lapierre LR, De Magalhaes Filho CD, McQuary PR, Chu CC, Visvikis O, Chang JT et al. The TFEB orthologue HLH-30 regulates autophagy and modulates longevity in Caenorhabditis elegans. Nat Commun 2013; 4: 2267.

76 Melendez A, Talloczy Z, Seaman M, Eskelinen EL, Hall DH, Levine B. Autophagy genes are essential for dauer development and life-span extension in C. elegans. Science 2003; 301: 1387-1391.

77 Sheaffer KL, Updike DL, Mango SE. The target of rapamycin pathway antagonizes pha-4/FoxA to control development and aging. Curr Biol 2008; 18: 1355-1364.

78 Seo K, Choi E, Lee D, Jeong DE, Jang SK, Lee SJ. Heat shock factor 1 mediates the longevity conferred by inhibition of TOR and insulin/IGF-1 signaling pathways in C. elegans. Aging Cell 2013; 12: 1073-1081.

79 Dillin A, Hsu AL, Arantes-Oliveira N, Lehrer-Graiwer J, Hsin H, Fraser AG et al. Rates of behavior and aging specified by mitochondrial function during development. Science 2002; 298: 2398-2401.

80 Tsang WY, Sayles LC, Grad LI, Pilgrim DB, Lemire BD. Mitochondrial respiratory chain deficiency in Caenorhabditis elegans results in developmental arrest and increased life span. J Biol Chem 2001; 276: 32240-32246.

81 Ventura N, Rea S, Henderson ST, Condo I, Johnson TE, Testi R. Reduced expression of frataxin extends the lifespan of Caenorhabditis elegans. Aging Cell 2005; 4: 109-112.

82 Feng J, Bussiere F, Hekimi S. Mitochondrial electron transport is a key determinant of life span in Caenorhabditis elegans. Dev Cell 2001; 1: 633-644.

83 Yang W, Hekimi S. A mitochondrial superoxide signal triggers increased longevity in Caenorhabditis elegans. PLOS Biol 2010; 8: e1000556.

84 Wong A, Boutis P, Hekimi S. Mutations in the clk-1 gene of Caenorhabditis elegans affect developmental and behavioral timing. Genetics 1995; 139: 1247-1259.

85 Braeckman BP, Houthoofd K, De Vreese A, Vanfleteren JR. Apparent uncoupling of energy production and consumption in longlived Clk mutants of Caenorhabditis elegans. Curr Biol 1999; 9: 493-496.

86 Ewbank JJ, Barnes TM, Lakowski B, Lussier M, Bussey H, Hekimi S. Structural and functional conservation of the Caenorhabditis elegans timing gene clk-1. Science 1997; 275: 980-983.

87 Lee SS, Lee RY, Fraser AG, Kamath RS, Ahringer J, Ruvkun G. A systematic RNAi screen identifies a critical role for mitochondria in $C$. elegans longevity. Nat Genet 2003; 33: 40-48.

88 Hansen M, Hsu AL, Dillin A, Kenyon C. New genes tied to endocrine, metabolic, and dietary regulation of lifespan from a Caenorhabditis elegans genomic RNAi screen. PLoS Genet 2005; 1: $119-128$.

89 Ray A, Martinez BA, Berkowitz LA, Caldwell GA, Caldwell KA. Mitochondrial dysfunction, oxidative stress, and neurodegeneration elicited by a bacterial metabolite in a $C$. elegans Parkinson's model. Cell Death Dis 2014; 5: e984.

90 Kayser EB, Morgan PG, Sedensky MM. GAS-1: a mitochondrial protein controls sensitivity to volatile anesthetics in the nematode Caenorhabditis elegans. Anesthesiology 1999; 90: 545-554.

91 Kondo M, Senoo-Matsuda N, Yanase S, Ishii T, Hartman PS, Ishii N. Effect of oxidative stress on translocation of DAF-16 in oxygen-sensitive mutants, mev-1 and gas-1 of Caenorhabditis elegans. Mech Ageing Dev 2005; 126: 637-641.

92 Chung KK, David KK. Emerging roles of nitric oxide in neurodegeneration. Nitric Oxide 2010; 22: 290-295.
93 Franco MC, Ye Y, Refakis CA, Feldman JL, Stokes AL, Basso M et al. Nitration of Hsp90 induces cell death. Proc Natl Acad Sci USA 2013; 110: E1102-E1111.

94 Schildknecht S, Gerding HR, Karreman C, Drescher M, Lashuel HA, Outeiro TF et al. Oxidative and nitrative alpha-synuclein modifications and proteostatic stress: implications for disease mechanisms and interventions in synucleinopathies. J Neurochem 2013; 125: 491-511.

95 Akbar M, Essa MM, Daradkeh G, Abdelmegeed MA, Choi Y, Mahmood $\mathrm{L}$ et al. Mitochondrial dysfunction and cell death in neurodegenerative diseases through nitroxidative stress. Brain Res 2016; 1637: 34-55.

$96 \mathrm{Ng}$ LF, Gruber J, Cheah IK, Goo CK, Cheong WF, Shui G et al. The mitochondria-targeted antioxidant MitoQ extends lifespan and improves healthspan of a transgenic Caenorhabditis elegans model of Alzheimer disease. Free Radic Biol Med 2014; 71: 390-401.

97 Miquel E, Cassina A, Martinez-Palma L, Souza JM, Bolatto C, Rodriguez-Bottero $S$ et al. Neuroprotective effects of the mitochondriatargeted antioxidant MitoQ in a model of inherited amyotrophic lateral sclerosis. Free Radic Biol Med 2014; 70: 204-213.

98 Springer W, Hoppe T, Schmidt E, Baumeister R. A Caenorhabditis elegans Parkin mutant with altered solubility couples alpha-synuclein aggregation to proteotoxic stress. Hum Mol Genet 2005; 14: 3407-3423.

99 Ved R, Saha S, Westlund B, Perier C, Burnam L, Sluder A et al. Similar patterns of mitochondrial vulnerability and rescue induced by genetic modification of alpha-synuclein, parkin, and DJ-1 in Caenorhabditis elegans. J Biol Chem 2005; 280: 42655-42668.

100 Wang H, Lim PJ, Yin C, Rieckher M, Vogel BE, Monteiro MJ. Suppression of polyglutamine-induced toxicity in cell and animal models of Huntington's disease by ubiquilin. Hum Mol Genet 2006; 15: 1025-1041.

101 Wang H, Lim PJ, Karbowski M, Monteiro MJ. Effects of overexpression of huntingtin proteins on mitochondrial integrity. Hum Mol Genet 2009; 18: 737-752.

102 Chan DC. Mitochondria: dynamic organelles in disease, aging, and development. Cell 2006; 125: 1241-1252.

103 Ron D, Walter P. Signal integration in the endoplasmic reticulum unfolded protein response. Nat Rev Mol Cell Biol 2007; 8: 519-529.

104 Walter P, Ron D. The unfolded protein response: from stress pathway to homeostatic regulation. Science 2011; 334: 1081-1086.

105 Doyle KM, Kennedy D, Gorman AM, Gupta S, Healy SJ, Samali A. Unfolded proteins and endoplasmic reticulum stress in neurodegenerative disorders. J Cell Mol Med 2011; 15: 2025-2039.

106 Sano R, Reed JC. ER stress-induced cell death mechanisms. Bioc him Biophys Acta 2013; 1833: 3460-3470.

107 Chen D, Thomas EL, Kapahi P. HIF-1 modulates dietary restrictionmediated lifespan extension via IRE-1 in Caenorhabditis elegans. PLoS Genet 2009; 5: e1000486.

108 Henis-Korenblit S, Zhang P, Hansen M, McCormick M, Lee SJ, Cary M et al. Insulin/IGF-1 signaling mutants reprogram ER stress response regulators to promote longevity. Proc Natl Acad Sci USA 2010; 107: 9730-9735.

109 Safra M, Ben-Hamo S, Kenyon C, Henis-Korenblit S. The ire-1 ER stress-response pathway is required for normal secretory-protein metabolism in C. elegans. J Cell Sci 2013; 126: 4136-4146.

110 Kieran D, Woods I, Villunger A, Strasser A, Prehn JH. Deletion of the $\mathrm{BH} 3-$ only protein puma protects motoneurons from ER stress-induced apoptosis and delays motoneuron loss in ALS mice. Proc Natl Acad Sci USA 2007; 104: 20606-20611.

111 Matus S, Lopez E, Valenzuela V, Nassif M, Hetz C. Functional contribution of the transcription factor ATF4 to the pathogenesis of amyotrophic lateral sclerosis. PLOS ONE 2013; 8: e66672.

112 Matus S, Valenzuela V, Medinas DB, Hetz C. ER dysfunction and protein folding stress in ALS. Int J Cell Biol 2013; 2013: 674751.

113 Vaccaro A, Patten SA, Aggad D, Julien C, Maios C, Kabashi E et al. Pharmacological reduction of ER stress protects against TDP-43 neuronal toxicity in vivo. Neurobiol Dis 2013; 55: 64-75.

114 Ray A, Zhang S, Rentas C, Caldwell KA, Caldwell GA. RTCB-1 mediates neuroprotection via XBP-1 mRNA splicing in the unfolded protein response pathway. J Neurosci 2014; 34: 16076-16085.

115 Zimprich A, Biskup S, Leitner P, Lichtner P, Farrer M, Lincoln S et al. Mutations in LRRK2 cause autosomal-dominant parkinsonism with pleomorphic pathology. Neuron 2004; 44: 601-607.

$116 \mathrm{Ng} \mathrm{CH}$, Mok SZ, Koh C, Ouyang X, Fivaz ML, Tan EK et al. Parkin protects against LRRK2 G2019S mutant-induced dopaminergic neurodegeneration in Drosophila. J Neurosci 2009; 29: 11257-11262. 
117 Dauer W, Ho CC. The biology and pathology of the familial Parkinson's disease protein LRRK2. Mov Disord 2010; 25(Suppl 1): S40-S43.

118 Samann J, Hegermann J, von Gromoff E, Eimer S, Baumeister R, Schmidt E. Caenorhabditits elegans LRK-1 and PINK-1 act antagonistically in stress response and neurite outgrowth. J Biol Chem 2009; 284 : 16482-16491.

119 Yuan Y, Cao P, Smith MA, Kramp K, Huang Y, Hisamoto N et al. Dysregulated LRRK2 signaling in response to endoplasmic reticulum stress leads to dopaminergic neuron degeneration in C. elegans. PloS ONE 2011; 6: e22354.

120 Denzel MS, Storm NJ, Gutschmidt A, Baddi R, Hinze Y, Jarosch E et al. Hexosamine pathway metabolites enhance protein quality control and prolong life. Cell 2014; 156: 1167-1178. (c) (i) (s) $\ominus$ This work is licensed under a Creative Commons Attribution-NonCommercial-NoDerivs 4.0 International License. The images or other third party material in this article are included in the article's Creative Commons license, unless indicated otherwise in the credit line; if the material is not included under the Creative Commons license, users will need to obtain permission from the license holder to reproduce the material. To view a copy of this license, visit http://creativecommons.org/licenses/by-nc-nd/4.0/ 